\title{
New approach to teaching matrix optics
}

Henri Arsenault

Henri H. Arsenault, "New approach to teaching matrix optics," Proc. SPIE 1603, Education in Optics, (1 March 1992); doi: 10.1117/12.57837 


\title{
A NEW APPROACH TO TEACHING MATRIX OPTICS
}

\author{
Henri H. Arsenault \\ Centre d'optique, photonique et lasers, Université Laval, Québec, Qc, Canada, G1K 7P4
}

\begin{abstract}
The classical $2 \times 2$ matrix approach to geometrical optics is very limited for practical use, because fundamental decompositions of system matrices do not yield matrices with physically significant properties. The use of permuted matrices proposed by us previously (J. Opt. Soc. Am. 73, 1350-1359) yields a much more powerful representation that is more useful for teaching from beginning undergraduate to advanced graduate levels. The matrices of the previous theory are still valid, but when they are treated in what might be called the focal plane representation, the matrices obtained by the LDU decomposition have a simple and direct physical meaning. The relationship between the older matrix theory and this one is analogous to the relationship between the Descartes and the Newton formalisms of geometrical optics: matrix components are simplified by measuring all distances from the foci. This facilitates synthesis problems, for which the standard approach is not well adapted. In addition to simple applications like lenses, mirrors and diopters, the theory can be applied to more complex cases like lenslike media, resonators, Fourier transform systems and phase-conjugate mirrors. This theory can be directly generalized for nonsymmetrical systems using a $4 \times 4$ matrix formalism. The other theory, where distances are measured from the principal planes, cannot be generalized for nonsymmetrical systems having no principal planes.
\end{abstract}

\section{INTRODUCTION}

The teaching of geometrical optics is often uses a matrix representation which is useful, because the manipulation of matrices is well-known and relatively simple. The matrix representation is based on the linear relationship between the spatial coordinates of a ray propagating between an input and an output plane:

$$
\begin{aligned}
& x^{\prime}=A x+B \xi \\
& \xi^{\prime}=C x+D \xi
\end{aligned}
$$

where $\mathrm{x}$ and $\mathrm{x}^{\prime}$ are the spatial coordinates and $\xi$ and $\xi^{\prime}$ are the direction cosines at the input and output planes. These equations may be expressed in matrix form

$$
\left[\begin{array}{l}
\mathbf{x}^{\prime} \\
\xi^{\prime}
\end{array}\right]=\left[\begin{array}{ll}
\mathrm{A} & \mathrm{B} \\
\mathrm{C} & \mathrm{D}
\end{array}\right]\left[\begin{array}{l}
\mathrm{x} \\
\xi
\end{array}\right]=\mathbf{M}\left[\begin{array}{l}
\mathbf{x} \\
\xi
\end{array}\right]
$$

The problem with this representation is that the decomposition of a system matrix $\mathbf{M}$ into elements that are fundamental from the matrix theory point of view are not fundamental from the Optics point of view. This is not very good from a pedagogical point of view, and limits the usefulness of the matrix representation for teaching purposes. From the Optics point of view, the fundamental quantities are the principal planes and the foci of the system. The fundamental matrix decomposition is the so-called LDU decomposition,

$$
\mathbf{M}=\mathbf{L D U}
$$

where $\mathbf{L}, \mathbf{D}$, and $\mathbf{U}$ are respectively a lower triangular matrix, a diagonal matrix, and an upper-diagonal matrix. We have shown ${ }^{1}$ that the LDU elements of a transfer matrix $M$ respectively represent a lens passage through a lens, a scaling of the coordinates, and a free-space propagation. These parameters are not usually the ones that are of interest in optical systems, so we searched for a better representation.

Note that in the matrix theory the propagation through the system is from left to right, whereas in the illustrations, we shall maintain the tradition of having light travel from right to left.

\section{A DIFFERENT MATRIX REPRESENTATION}

We found that a slight modification of the representation yielded a much more useful representation. All we have to do is use the row-permuted system matrix $\mathbf{P M}$ instead of the system matrix itself, where $\mathbf{P}$ is the permutation matrix

$$
\mathbf{P}=\left[\begin{array}{ll}
0 & 1 \\
1 & 0
\end{array}\right]
$$


This can be done from existing equations by multiplying both sides of Eq. (3) by the permutation matrix P.For a general system matrix

$$
\mathbf{M}=\left[\begin{array}{ll}
\text { A } & B \\
\text { C } & D
\end{array}\right]
$$

this yields

$$
\mathbf{P}\left[\begin{array}{l}
\mathbf{x}^{\prime} \\
\xi^{\prime}
\end{array}\right]=\mathbf{P M}=\mathbf{L D U}=\left[\begin{array}{cc}
1 & 0 \\
\mathrm{~A} / \mathrm{C} & 1
\end{array}\right]\left[\begin{array}{cc}
\mathrm{C} & 0 \\
0 & \mathrm{~B}-\mathrm{AD} / \mathrm{C}
\end{array}\right]\left[\begin{array}{cc}
1 & \mathrm{D} / \mathrm{C} \\
0 & 1
\end{array}\right]
$$

An equivalent (and more physical) representation is to represent the system matrix $\mathbf{M}$ as an anti-diagonal matrix with an upper-diagonal matrix on each side. It is easy to see that

$$
\mathbf{M}=\mathbf{U}_{1} \mathbf{A} \mathbf{U}_{2}=\left[\begin{array}{ll}
1 & \mathrm{a} \\
0 & 1
\end{array}\right]\left[\begin{array}{cc}
0 & \mathrm{f} \\
-1 / \mathrm{f} & 0
\end{array}\right]\left[\begin{array}{ll}
1 & \mathrm{~b} \\
0 & 1
\end{array}\right]
$$

where $a=A / C, b=D / C$ and $f=-1 / C$. In this representation, each matrix of the decomposition has a direct physical meaning. First notice that the antidiagonal matrix A corresponds to propagation between the focal planes of the system; so the two matrices $\mathbf{U}_{1}$ and $\mathbf{U}_{2}$ correspond to propagations between the entrance and exit planes and the foci. Remembering that in this matrix theory, propagation goes from left to right, the general decomposition corresponds to propagation from the entrance plane through a distance $b$ to the front focal plane, followed by propagation through the system to the back focal plane, followed by propagation from the back focal plane to the exit plane through a distance a.

So we see that this decomposition, that we have called the focal plane representation, immediately gives us the equivalent focal length $f$ of the system as well as the positions of the focal planes with respect to the input and output planes.

Of course the principal planes are at distances $f$ from the focal planes. The matrix for propagation between the principal planes is

$$
\mathbf{M}=\left[\begin{array}{cc}
1 & 0 \\
1 / \mathrm{f} & 1
\end{array}\right]
$$

which of course is also the matrix representation of a thin lens.

The sign conventions are the same as for the Newton $\mathrm{S}_{\mathrm{O}} \mathrm{S}_{\mathrm{i}}=\mathrm{f}^{2}$ representation. In summary,

1) If $f$ is positive, the foci are located outside the principal planes, and if $f$ is negative, the foci are located inside those planes.

2) If $b$ is positive, then the focus $F_{1}$ is on the right side of the input plane, and if $b$ is negative, $F_{1}$ is on the left side of the input plane.

3) If a is positive, then the focus $F_{2}$ is on the right side of the input plane, and if a is negative, $F_{1}$ is on the left side of the input plane.

4) The distances between the principal planes and the focal planes are $K_{1}=b+f$ and $K_{2}=a+f$.

One may ask: why not use directly the LDU decomposition of the original matrix $\mathbf{M}$ istead of the permuted matrix PM? The answer is the following: the LDU decomposition for the propagation from an input plane at a distance $d_{1}$ from a thin lens to an output plane at a distance $d_{2}$ after the lens is

$$
\mathbf{M}=\left[\begin{array}{cc}
1 & 0 \\
\frac{-1}{f-d_{2}} & 1
\end{array}\right]\left[\begin{array}{cc}
\frac{f-d_{2}}{f} & 0 \\
0 & \frac{f}{f-d_{2}}
\end{array}\right]\left[\begin{array}{cc}
1 \frac{d_{1} f+d_{2} f-d_{1} d_{2}}{f-d_{2}} \\
0 & 1
\end{array}\right]
$$

We see that if $d_{2}=f$, the term in the matrix on the left become infinite, so this representation does not allow the calculation of propagations from the foci of the system. On the other hand, the permuted decomposition has no singularities, except for the impossible case $f=0$. As it turns out, the simplest representation of a lens is for the propagation between its foci, becauase in the new representation, we see by setting $a=b=0$ in Eq, (8) that the focal plane representation of a lens is 


$$
\mathbf{L}=\left[\begin{array}{cc}
0 & \mathbf{f} \\
-1 / f & 0
\end{array}\right]
$$

and we see that for any optical system, the operation of diagonalization of the system matrix corresponds to reducing the system to its focal length representation.

It is important to note that the system matrix $\boldsymbol{M}$ is still the same matrix as in the old theory. This is very useful for pedagogical purposes, because we have the best of both worlds: the old matrix discussions in textbooks is still valid, and our new approach adds to this knowledge (in addition to being easier to use).

We now consider some simple examples that show how this approach can be used both for theoretical purposes and to solve practical problems.

\section{TWO THIN LENSES}

To illustrate the pedagogical usefulness of this approach, let us calculate the cardinal points of two thin lenses with their foci separated by a distance $d$ as illustrated in Fig. 1. Starting from the front focal plane of the first lens $L_{1}$ and finishing at the back focal plane of the second lens, the system matrix is

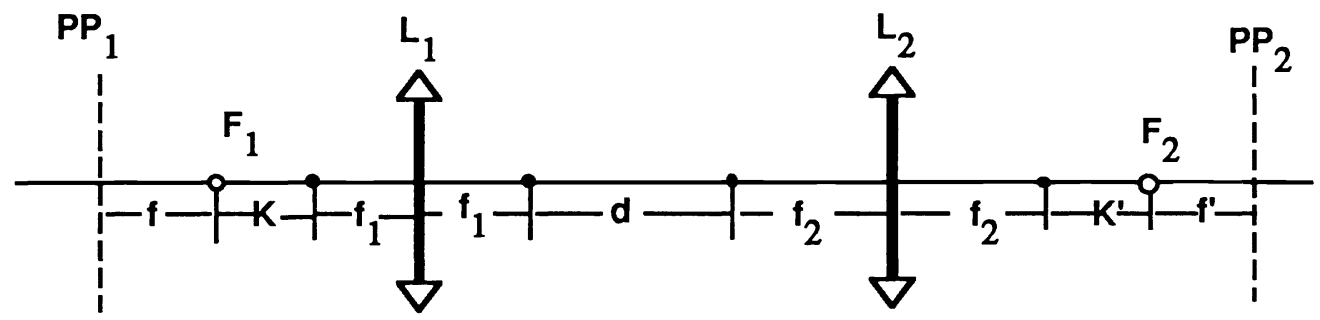

Figure 1: Two-lens system

$$
\mathbf{P M}=\left[\begin{array}{cc}
-1 / \mathrm{f}_{2} & 0 \\
0 & \mathrm{f}_{2}
\end{array}\right]\left[\begin{array}{cc}
1 & \mathrm{~d} \\
0 & 1
\end{array}\right]\left[\begin{array}{cc}
0 & \mathrm{f}_{1} \\
-1 / \mathrm{f}_{1} & 0
\end{array}\right]=\left[\begin{array}{cc}
\mathrm{d} / \mathrm{f}_{1} \mathrm{f}_{2} & -\mathrm{f}_{1} / \mathrm{f}_{2} \\
-\mathrm{f}_{2} / \mathrm{f}_{1} & 0
\end{array}\right]
$$

where the first matrix on the left represents the propagation between the two focal planes of lens $\mathrm{L}_{1}$, the central matrix is the propagation between the two foci, and the matrix on the left is the permuted matrix for the propagation between the two foci of lens $\mathrm{L}_{2}$. The LDU decomposition of this matrix is

$$
\mathbf{P M}=\left[\begin{array}{cc}
1 & 0 \\
-\mathrm{f}_{2}^{2} / \mathrm{d} & 1
\end{array}\right]\left[\begin{array}{cc}
\mathrm{d} / \mathrm{f}_{1} \mathrm{f}_{2} & 0 \\
0 & -\mathrm{f}_{1} \mathrm{f}_{2} / \mathrm{d}
\end{array}\right]\left[\begin{array}{cc}
1 & -\mathrm{f}_{1}{ }^{2} / \mathrm{d} \\
0 & 1
\end{array}\right]
$$

and applying the permutation operator $\mathbf{P}$ to the matrix on the left yields

$$
\mathbf{M}=\left[\begin{array}{cc}
1 & -\mathrm{f}_{2}^{2} / \mathrm{d} \\
0 & 1
\end{array}\right]\left[\begin{array}{cc}
\mathrm{d} / \mathrm{f}_{1} \mathrm{f}_{2} & 0 \\
0 & -\mathrm{f}_{1} \mathrm{f}_{2} / \mathrm{d}
\end{array}\right]\left[\begin{array}{cc}
1 & -\mathrm{f}_{1}^{2} / \mathrm{d} \\
0 & 1
\end{array}\right]
$$

The interpretation of this expression produces new and simpler relations than those commonly used for the positions of the cardinal points of a two-lens system. Since the matrix in the middle contains the equivalent focal length and the two others contain the distances to the foci of the system, the focal length of the combined system is

$$
f^{\prime}=-f_{1} f_{2} / d
$$

and that the distances between the foci of the combined system and the foci of the individual lenses is

$$
\begin{aligned}
& K^{\prime}=f_{2}^{2} / d \\
& K=-f_{1}{ }^{2} / d .
\end{aligned}
$$

The principal planes, of course, are located at distances $\pm f^{\prime}$ from the system foci. 
There are a few things that are worth pointing out at this point. First, we see that the expressions (12) - (14) for the focal length and the positions of the foci appear directly in the LDU decomposition; second, the expressions for the positions of the foci are simpler that those that are commonly used (measured from the lenses). The conclusion is important and is also valid for more complex systems: it is simpler if everything is always measured from the foci of the lenses instead of from the lenses themselves.

In the matrix theory usually taught in textbooks, the student must learn not only the matrix representation, but also expressions for the cardinal points. With this approach, this is not necessary, because the quantities of interest appear directly as matrix elements. And if the professor or student would rather work with the mathematical expressions, expressions such as (16) and (17) are easier to remember than the standard expressions with distances measured from the lenses, because all the expressions are of the form of Newton's equation $S_{0} S_{i}=f^{2}$.

\section{THREE LENS SYSTEM}

To illustrate this, consider a three-lens system as shown in Fig. 2.

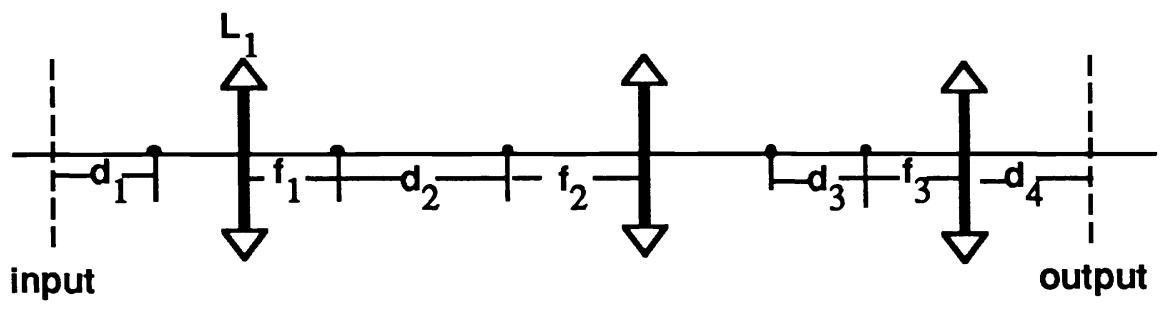

Figure 2: Three-lens system

The system matrix for propagation from an arbitrary plane at a distance $d_{1}$ to the left of the front focal plane of the first lens to an arbitrary plane at a distance $d_{2}$ to the right of the back focal plane of the third lens is

$$
\mathbf{M}=\frac{1}{\mathrm{f}_{1} \mathrm{f}_{2} \mathrm{f}_{3}}\left[\begin{array}{cc}
\mathrm{d}_{4} \mathrm{f}_{2}^{2}+\mathrm{d}_{2} \mathrm{f}_{3}^{2}-\mathrm{d}_{2} \mathrm{~d}_{3} \mathrm{f}_{4} & -\mathrm{d}_{1} \mathrm{~d}_{2} \mathrm{~d}_{3} \mathrm{~d}_{4}+\mathrm{d}_{1} \mathrm{~d}_{4} \mathrm{f}_{2}^{2}+\mathrm{d}_{1} \mathrm{~d}_{2} \mathrm{f}_{3}^{2}+\mathrm{d}_{3} \mathrm{~d}_{4} \mathrm{f}_{1}^{2}-\mathrm{f}_{1}^{2} \mathrm{f}_{3}^{2} \\
\mathrm{f}_{2}^{2}-\mathrm{d}_{2} \mathrm{~d}_{3} & -\mathrm{d}_{1} \mathrm{~d}_{2} \mathrm{~d}_{3}+\mathrm{d}_{1} \mathrm{f}_{2}^{2}+\mathrm{d}_{3} \mathrm{f}_{1}^{2}
\end{array}\right]
$$

and the permuted LDU decomposition yields

$$
\mathbf{M}=\left[\begin{array}{cc}
1 & \mathrm{~d}_{4}+\frac{\mathrm{d}_{2} \mathrm{f}_{3}^{2}}{\mathrm{f}_{2}^{2}-\mathrm{d}_{2} \mathrm{~d}_{3}} \\
0 & 1
\end{array}\right]\left[\begin{array}{cc}
0 & \frac{-\mathrm{f}_{1} \mathrm{f}_{2} \mathrm{f}_{3}}{\mathrm{f}_{2}^{2}-\mathrm{d}_{2} \mathrm{~d}_{3}} \\
\frac{\mathrm{f}_{2}^{2}-\mathrm{d}_{2} \mathrm{~d}_{3}}{\mathrm{f}_{1} \mathrm{f}_{2} \mathrm{f}_{3}} & 0
\end{array}\right]\left[\begin{array}{cc}
1 & \mathrm{~d}_{1}+\frac{\mathrm{d}_{3} \mathrm{f}_{1}^{2}}{\mathrm{f}_{2}^{2}-\mathrm{d}_{2} \mathrm{~d}_{3}} \\
0 & 1
\end{array}\right]
$$

which immediately tells us that the equivalent focal length is equal to

$$
f=\frac{f_{1} f_{2} f_{3}}{f_{2}^{2}-d_{2} d_{3}}
$$

and that the front focal plane is at a distance

$$
\mathrm{b}=\mathrm{d}_{1}+\frac{\mathrm{d}_{3} \mathrm{f}_{1}^{2}}{\mathrm{f}_{2}^{2}-\mathrm{d}_{2} \mathrm{~d}_{3}}
$$

from the input plane, and that the back focal plane is at a distance 


$$
a=d_{4}+\frac{d_{2} f_{3}^{2}}{f_{2}^{2}-d_{2} d_{3}}
$$

from the output plane. If the input planes had been taken at the front focal plane of the first lens and the output plane at the back focal plane of the last lens, the expressions would be the same except that $\mathrm{d}_{1}$ and $\mathrm{d}_{4}$ would be equal to zero.

\section{PRACTICAL PROBLEMS}

The old matrix approach is not very useful for solving practical geometrical optics problems, and students usually have to forego the matrix approach to solve the problems they are assigned. On the other hand this approach is quite powerful, and can be used directly to solve relatively complicated problems. To illustrate this, consider the following moderately complex problem of a focal length doubler for a camera. I have given this problem (with real numbers) on an exam to second-year Physics students trained with the old methods, and most of them were unable to find the answer. With the new method, it can be done in minutes. Here we do the more tedious calculation using variables.

A camera with a lens $L_{1}$ with a focal length $f_{1}$ is to have its focal length doubled by means of a lens $L_{2}$. Lens $L_{2}$ replaces lens $L_{1}$, and lens $L_{1}$ must be placed at an unknown distance $d$ in front of lens $L_{2}$. What is the focal length of $L_{2}$ and the distance $\mathrm{d}$ between the two lenses?

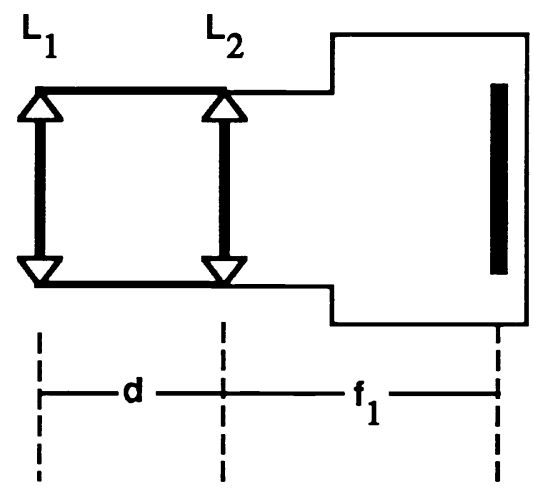

Figure 3: Focal length doubler

For a first course in geometrical optics, this problem is relatively fastidious to solve, but with our matrix approach the solution is quick and easy. The propagation between the two lenses is given by the matrix product

$$
M=\left[\begin{array}{cc}
1 & 0 \\
-1 / f_{2} & 1
\end{array}\right]\left[\begin{array}{ll}
1 & d \\
0 & 1
\end{array}\right]\left[\begin{array}{cc}
1 & 0 \\
-1 / f_{1} & 1
\end{array}\right]=\left[\begin{array}{cc}
1-d / f_{1} & d \\
d / f_{1} f_{2}-1 / f_{1}-1 / f_{2} & 1-d / f_{2}
\end{array}\right]
$$

The now familiar decomposition yields

$$
M=\left[\begin{array}{cc}
1 & \frac{f_{2}\left(f_{1}-d\right)}{d-f_{1}-f_{2}} \\
0 & 1
\end{array}\right]\left[\begin{array}{cc}
0 & \frac{f_{1} f_{2}}{f_{1}+f_{2}-d} \\
\frac{-f_{1}-f_{2}+d}{f_{1} f_{2}} & 0
\end{array}\right]\left[\begin{array}{cc}
1 & \frac{f_{1}\left(f_{2}-d\right)}{d-f_{1}-f_{2}} \\
0 & 1
\end{array}\right]
$$

Now the focal length contained in the middle matrix must be equal to $2 \mathrm{f}_{1}$, so

$$
\frac{\mathrm{f}_{1} \mathrm{f}_{2}}{\mathrm{f}_{1}+\mathrm{f}_{2}-\mathrm{d}}=2 \mathrm{f}_{1}
$$

and the back focal plane of the system must be unchanged on the film, that is at a distance $f_{1}$ behind lens $L_{2}$, and this is the distance on the matrix on the left side, so

$$
\frac{f_{2}\left(f_{1}-d\right)}{d-f_{1}-f_{2}}=-f_{1}
$$


These two equations are easily solved to yield the solution

$$
\begin{aligned}
f_{2} & =-f_{1} \\
d & =f_{1} / 2 .
\end{aligned}
$$

For this problem, as for most geometrical problems solved using this approach, the solution is straightforward, and involves only or mainly decomposing the transposed matrix into its LDU decomposition.

\section{ADVANCED PROBLEMS}

The matrix approach described above can be applied to more complex systems such as thick lenses, lenslike media, symmetrical mirrors and resonators, phase-conjugate mirrors, cylindrical lenses and mirrors, and may be generalized to include non-symmetrical optics like thick cylindrical diopters and lenses with arbitrary orientations.

As a matter of fact, it was the study of the geometrical optics of nonsymmetrical systems and the realization that in general, such systems systems do not have principal planes, that led us to develop a matrix theory for such systems of which the theory presented here is a special case.

Space limitations do not allow the full description of all those systems, and the interested reader is referred to the bibliography for details. We present here only a few cases of educational interest.

\section{SPHERICAL DIOPTER}

An important case is the spherical diopter, whose transfer matrix is easily shown ${ }^{2}$ to be equal to

$$
\mathbf{M}=\left[\begin{array}{cc}
1 & 0 \\
\frac{\mathrm{n}_{1}-\mathrm{n}_{2}}{\mathrm{R}} & 1
\end{array}\right]
$$

where $\mathrm{R}$ is the radius of the diopter, and $\mathrm{n}_{1}$ and $\mathrm{n}_{2}$ are respectively the index of refraction of the input and of the output media. The permuted LDU decomposition of this matrix

$$
\mathbf{M}=\left[\begin{array}{cc}
1 & \frac{-R}{n_{2}-n_{1}} \\
0 & 1
\end{array}\right]\left[\begin{array}{cc}
0 & \frac{R}{n_{2}-n_{1}} \\
\frac{n_{1}-n_{2}}{R} & 0
\end{array}\right]\left[\begin{array}{cc}
1 & \frac{-R}{n_{2}-n_{1}} \\
0 & 1
\end{array}\right]
$$

immediately yields the two focal lengths of the diopter

$$
\begin{aligned}
\mathrm{f}_{1} & =\frac{\mathrm{n}_{1} R}{\mathrm{n}_{2}-\mathrm{n}_{1}} \\
\mathrm{fd}_{2} & =\frac{\mathrm{n}_{2} R}{\mathrm{n}_{2}-\mathrm{n}_{1}}
\end{aligned}
$$

taking into account that the distances must be multiplied by the index of refraction of the medium.

\section{THICK LENS}

The cardinal points of a thick lens may be obtained in a straightforward manner by combining two diopters. Consider the general case of a thick lens of index $n_{2}$ immersed in media of $n_{1}$ and $n_{2}$ on the entrance and exit sides respectively.

It is easily calculated from Eq. (25) that the propagation from the front focal plane of the first diopter to the back focal plane of the second diopter constituting the thick lens is given by the matrix

$$
\mathbf{M}=\left[\begin{array}{cc}
\frac{\mathrm{R}_{2}\left(\mathrm{n}_{2}-\mathrm{n}_{1}\right)}{\mathrm{R}_{1}\left(\mathrm{n}_{2}-\mathrm{n}_{3}\right.} & 0 \\
\frac{\left(\mathrm{n}_{3}-\mathrm{n}_{2}\right)\left(\mathrm{n}_{2}-\mathrm{n}_{1}\right)}{\mathrm{R}_{1} \mathrm{R}_{2}}\left(\frac{\mathrm{d}}{\mathrm{n}_{2}}-\frac{\mathrm{R}_{1}}{\mathrm{n}_{2}-\mathrm{n}_{1}}-\frac{\mathrm{R}_{2}}{\mathrm{n}_{3}-\mathrm{n}_{2}}\right) & \frac{\mathrm{R}_{1}\left(\mathrm{n}_{2}-\mathrm{n}_{3}\right)}{\mathrm{R}_{2}\left(\mathrm{n}_{1}-\mathrm{n}_{2}\right)}
\end{array}\right]
$$

whose LDU decomposition, after row permutation yields 


$$
\mathbf{M}=\left[\begin{array}{cc}
1 & \frac{f R_{2}\left(n_{1}-n_{2}\right)}{R_{1}\left(n_{2}-n_{3}\right)} \\
0 & 1
\end{array}\right]\left[\begin{array}{cc}
0 & f \\
-1 / f & 0
\end{array}\right]\left[\begin{array}{cc}
1 & \frac{f R_{1}\left(n_{2}-n_{3}\right)}{R_{1}\left(n_{1}-n_{2}\right)} \\
0 & 1
\end{array}\right]
$$

with

$$
\frac{1}{\mathrm{f}}=\frac{\mathrm{d}\left(\mathrm{n}_{3}-\mathrm{n}_{2}\right)\left(\mathrm{n}_{2}-\mathrm{n}_{1}\right)}{\mathrm{n}_{2} \mathrm{R}_{1} \mathrm{R}_{2}}-\frac{\mathrm{n}_{3}-\mathrm{n}_{2}}{\mathrm{R}_{2}}-\frac{\mathrm{n}_{2}-\mathrm{n}_{1}}{\mathrm{R}_{1}}
$$

This yields immediately the focal lengths of the lens, $f / n_{1}$ and $f / n_{3}$. The distances to the foci of the thick lens as measured from the entrance and exit points are given by the upper diagonal terms in the right and left matrices, multiplied by the index of refraction.

The above calculations are not trivial, because we are using theoretical values. If actual numbers are used in a practical problem, these calculations are almost trivial and can be calculated in minutes without any knowledge of the formulas.

\section{LENSLIKE MEDIA}

As a final and more advanced example, consider the propagation through a lenslike medium. This is a medium (such as an optical fiber) whose index of refraction varies quadratically with the distance $r$ from the optical axis

$$
\mathrm{n}=\mathrm{n}_{0}-\mathrm{n}_{2} \mathrm{r}^{2} / 2 \quad \mathrm{n}_{0}=\text { constant }
$$

The transfer matrix for such a system is

$$
\mathbf{M}=\left[\begin{array}{cc}
\cos \left[\mathrm{d}\left(\frac{\mathrm{n}_{2}}{\mathrm{n}_{0}}\right)^{2}\right] & \frac{1}{\left(\mathrm{n}_{0} \mathrm{n}_{2}\right)^{1 / 2}} \sin \left[\mathrm{d}\left(\frac{\mathrm{n}_{2}}{\mathrm{n}_{0}}\right)^{1 / 2}\right] \\
-\left(\mathrm{n}_{0} \mathrm{n}_{2}\right)^{1 / 2} \sin \left[\mathrm{d}\left(\frac{\mathrm{n}_{2}}{\mathrm{n}_{0}}\right)^{1 / 2}\right] & \cos \left[\mathrm{d}\left(\frac{\mathrm{n}_{2}}{\mathrm{n}_{0}}\right)^{2}\right]
\end{array}\right]
$$

When this matrix is factored into its permuted LDU form, the antidiagonal term yields the focal length

$$
f=\frac{1}{\left(n_{0} n_{2}\right)^{1 / 2} \sin \left[d\left(\frac{n_{2}}{n_{0}}\right)^{1 / 2}\right]}
$$

and the distances to the focal lengths from the input and output planes are the upper diagonal terms

$$
\mathrm{a}=\mathrm{b}=-\frac{1}{\left(\mathrm{n}_{0} \mathrm{n}_{2}\right)^{1 / 2}} \cot \left[\mathrm{d}\left(\frac{\mathrm{n}_{2}}{\mathrm{n}_{0}}\right)^{1 / 2}\right]
$$

Due to limited space, we refer the reader to the bibliography ${ }^{2}$ for other applications such as mirrors and resonators, phaseconjugate mirrors and applications to optical systems without rotational symmetry 3,4 , where the $2 \times 2$ matrices are replaced by $4 \times 4$ matrices.

\section{CONCLUSION}

The matrix approach to matrix optics presented here simultaneously builds upon the classical approach and yet modifies it radically. The matrices defined according to the previous approach are still valid, so it is not required that the professor and students forget previously acquired knowledge. Yet it profoundly modifies the approach to classical geometrical optics where the principal planes play a dominant role. In addition, the representation of an optical system as an antidiagonal matrix is simpler and corresponds to a more fundamental mathematical entity.

In the new approach, the principal planes are not even necessary: all the physical parameters needed and that appear in the decomposition refer to the foci of the system. This is more than a superficial accident, because when this approach is generalized to nonsymmetrical systems consisting for example of cylindrical lenses rotated by arbitrary angles, it is found that such systems do not in general have principal planes, but that they always have (line) foci. This shows that the focal plane approach is more fundamental than the approach based on principal planes. 
${ }^{1}$ H. H. Arsenault, Generalization of the principal plane concept in matrix optics, Am. J. Phys. 66, 397-399 (1980).

${ }^{2}$ H. H. Arsenault and B. Macukow, Factorixzation of the transfer matrix for symmetrical optical systems, J. Opt. Soc. Am. 73, 1350-1359 (1983).

${ }^{3}$ H. H. Arsenault and B. Macukow, Matrix decompositions for nonsymmetrical optical systems, J. Opt. Soc. Am. 73, 1360-1366 (1983).

${ }^{4}$ B. Macukow and H. H. Arsenault, Extension of the matrix theory for nonsymmetrical optical systems, J. Optics (Paris) $15,145-151$ (1984). 